\title{
Icosahedral frustrations ahead
}

\section{The experimenters have been more successful in finding new examples of icosahedral symmetry than the theoreticians have been at interpreting these exciting data.}

IN the two years since the discovery of an alloy of manganese and aluminium with icosahedral symmetry by Schechtman, Blech, Gratias and Cahn, most of us have become more knowledgeable about crystallography, but are still perplexed to know what icosahedral symmetry means. This is not to suggest that people have been idle. Making tiny samples of alloys other than the Schechtman alloy, but with the same unexpected symmetry, has been widely practised; electron and X-ray diffraction patterns with five- or tenfold symmetry have become familiar.

People with a feeling for solid geometry have become even more expert at telling how icosahedra may be assembled into the shapes called tricontrahedra, while people like Pauling, with a sixth sense for how crystals are constructed, say the ground is familiar. [Pauling's first statement of this case (Nature 317, 512; 1985), which has been disputed, will soon be followed by an elaboration.]

The difficulty remains that ordinary mortals have no way of visualizing the construction of an icosahedral crystal, called a quasicrystal because it must be aperiodic, by placing identical unit cells at the points in space defined by vectors such as those defining ordinary crystal lattices. There is no language in which to search for common ground.

Disappointingly, the most promising candidate has now been used to suggest that the task of telling the true structure of icosahedral crystals is virtually impossible. This is one conclusion of the latest account by Per Bak, of Brookhaven National Laboratory, of his way of regarding icosahedral crystals as the projections on three-dimensional real space of crystal lattices of a more familiar kind constructed in six dimensions (Phys. Rev. Lett. 56, $861 ; 1986$ ). Last year, Bak's account of how structures with only vestigial symmetry - such as the tiling of the two dimensional plane by two rhombi of different shape - may be related to more symmetrical structures in a higher dimension was one of the most stimulating features of the enquiry.

The argument has general interest. An icosahedron is a solid with 20 faces, each of which is an equilateral triangle. One way of visualizing the figure is to construct a pair of pentagonal pyramids and to bolt them together, one pentagonal base to the other, with a ring of ten equilateral triangles joined head to toe. There are twelve vertices, all geometrically equivalent, at which the corners of five triangles come together. Icosahedral symmetry is characterized by a total of 120 symmetry operations. For making crystals, icosahedra are unsatisfactory building blocks. For one thing, it is impossible to fill threedimensional space with them alone. For another, fivefold symmetry is literally incompatible with an underlying lattice structure of the Bravais type. And then there is no simple way of describing such a lattice, even if it could exist, in terms of integral multiples of some set of independent displacement vectors such as normally define the shape of a crystallographic unit cell.

The last difficulty, more than a mere annoyance, is most simply illustrated by a hexagonal lattice in two dimensions as in a sheet of carbon atoms from a graphite crystal. The simplest description is in terms of a pair of vectors of equal length making an angle of 60 degrees with each other. A little scribbling will show that this choice allows the construction of a twodimensional crystal lattice in which the smallest unit cell is a rhombus whose sides are three times as long as the basic hexagonal sides; each contains two complete hexagons and bits and pieces enough to make a third.

So why not find a more natural description reflecting the inherent hexagonal symmetry? The natural choice of basis vectors would be the set of three equal vectors defined in direction by the three hexagonal sides that meet at every vertex. An arbitrary choice must be made between the two orientations of triple vertices that occur, but the more serious difficulty is that the three vectors are not independent. (If their directions are chosen to point outwards from a triple vertex, their sum will be zero.) In effect, Bak's way of dealing with this difficulty is to pretend that the chosen vectors are in reality independent which, because there are three of them, means that they span three-dimensional space. As in an ordinary crystal, lattice points are then assumed to occur at all points represented by integral multiples of the basis vectors, and the two-dimensional hexagonal pattern will be found by cutting a suitable twodimensional plane through that threedimensional lattice.

Last year, Bak successfully applied this technique to the case of icosahedral symmetry, where the natural basis vectors are a set of six drawn from the centre of the figure to each of the vertices of a pentagonal pyramid chosen at random. (The other six vertices are reached by projecting these vectors backwards.) It does no harm if this set of basis vectors are visualized as orthogonal axes in six-dimensional space. The most general description of this crystal is by means of a function representing the likelihood that matter of a particular kind (manganese or aluminium atoms in the Schechtman case) will occur at some point in space, which must in turn be a periodic function reflecting the repetitiveness of lattice displacements. The underlying icosahedral symmetry is reflected in the way in which this density function can be represented by simple periodic functions such as sines and cosines; symmetrically equivalent Fourier coefficients are identical.

Getting from here to the real world is not so simple. The six-dimensional lattice has icosahedral symmetry, but real threedimensional space is not related to that pattern in a simple or even unique way. The trick is to classify the different ways in which the symmetry of the six-dimensional symmetry will show up on the threedimensional "hypersurface" which is the real world. The answer is that the positions of atoms in the real crystal will be determined by the intersection of a sequence of three-dimensional "surfaces" in six dimensions with the real threedimensional world (itself a hypersurface).

Two conclusions are arresting. First, Bak shows that it is possible to recover the now-familiar Penrose tiling of the twodimensional plane, and its threedimensional analogue, as special cases of his six-dimensional contruction. But he also shows that there are limitless other ways in which the appearence (in diffraction patterns) of icosahedral symmetry may be generated. The implications of that are stark. There is no "simple mathematical model" of the Schechtman alloy, and no alternative to the complete solution of six-dimensional crystal structures for those who wish to know where the atoms are in these quasi-crystals. But Pauling, who argues for the twinning of unit cells unable otherwise to fill space, will be glad to note that Bak offers the distortion of the "natural" unit cell as one way of looking at icosahedral symmetry. What nobody has yet considered seriously is the energetics of these odd forms.

John Maddox 